\title{
Gaia
}

Revue interdisciplinaire sur la Grèce archaïque

\section{À l'aube des villes antiques : vocabulaire de la cité et formes urbaines. - Introduction}

At the Dawn of Ancient Towns: Vocabulary of the City and Urban Forms.Introduction

\section{Arianna Esposito et Airton Pollini}

\section{(2) OpenEdition}

\section{Journals}

Édition électronique

URL : https://journals.openedition.org/gaia/736

DOI : $10.4000 /$ gaia.736

ISSN : 2275-4776

Éditeur

UGA Éditions/Université Grenoble Alpes

Édition imprimée

ISBN : 978-2-37747-199-7

ISSN : 1287-3349

\section{Référence électronique}

Arianna Esposito et Airton Pollini, «À l'aube des villes antiques : vocabulaire de la cité et formes

urbaines. - Introduction », Gaia [En ligne], 22-23 | 2020, mis en ligne le 30 juin 2020, consulté le 09

décembre 2021. URL : http://journals.openedition.org/gaia/736 ; DOI : https://doi.org/10.4000/gaia.

736

Ce document a été généré automatiquement le 9 décembre 2021.

Gaia. Revue interdisciplinaire sur la Grèce archaïque 


\section{À l'aube des villes antiques: vocabulaire de la cité et formes urbaines. - Introduction}

At the Dawn of Ancient Towns: Vocabulary of the City and Urban Forms.Introduction

\section{Arianna Esposito et Airton Pollini}

"Il Gran Kan possiede un atlante in cui sono raccolte

le mappe di tutte le città: quelle che elevano le loro mura su alte fondamenta, quelle che caddero in rovina

e furono inghiottite dalla sabbia, quelle che esisteranno un giorno e al cui posto ancora non s'aprono che le tane delle lepri. [...] Il catalogo delle forme è sterminato: finchè ogni forma non avrà trovato la sua città, nuove città continueranno a nascere. Dove le forme esauriscono le loro variazioni e si disfano, comincia la fine della città. »

(I. Calvino, Le città invisibili, Milan, Mondadori, 2001, p. 139-140)

« Le sens de ce mot [Cité] s'est presque entièrement effacé chez les modernes ; la plupart prennent une ville pour une cité et un bourgeois pour un citoyen. Ils ne savent pas que les maisons font la ville mais que les citoyens font la cité. »

(J.-J. Rousseau, Du contrat Social ou Principes du droit politique, liv. I, chap. VI, III, 1762) «Tout le monde sait ce qu'est une ville, à l'exception des experts. » 
(Horace M. Miner, The City in Modern Africa, New York/Londres, F. A. Praeger, 1967, p. 11)

1 Ce dossier est le premier résultat et, en même temps, une réflexion d'étape, d'une opération développée en partenariat par l'UMR 6298 ARTEHIS (programme «Polis, Apoikia, Vrbs, Oppidum, Ciuitas, Colonia: interprétations et réinterprétations de la cité antique de l'époque archaïque à l'Antiquité tardive») et l'UMR 7044 Archimède (opération «Naissance et mort de la cité ») ${ }^{1}$. Ce projet commun propose d'aborder la ville et la cité antiques en tant que concepts historiographiques et objets archéologiques, dans une perspective historique articulée suivant deux axes géographiques: la Méditerranée et l'Europe continentale (mondes grec, celtique, gaulois, italique, romain) $)^{2}$. Ce type d'approche comparatiste implique: géographiquement, un changement de l'échelle d'observation; chronologiquement, la prise en compte de la longue durée; enfin, du point de vue de la méthodologie, le recours à la transdisciplinarité, avec une collaboration entre les spécialistes des textes et de terrain. L'enjeu d'une définition, c'est aussi et en conséquence l'évaluation des théories qui la justifient. C'est bien cette discussion critique, à la fois du mot et de la phénoménologie archéologique, qui a animé nos ateliers, en croisant perspectives historique et archéologique : existe-t-il, dans les études sur l'Antiquité, un objet de recherche spécifique que nous pouvons appeler "cité»? Qu'est-ce qui définit par ailleurs une cité3? Quelles ont été les phases de sa formation, de son développement? Que sait-on aujourd'hui des premières expériences urbaines, des premières villes? Quels ont été les rythmes, les modalités et les projets urbanistiques de leur développement ? Dans quelle mesure peut-on parler « de la ville» au nom « des villes »?

2 Une analyse de la cité et de la ville antiques suppose d'emblée une définition opératoire". Or, comme l'affirmait déjà Numa Fustel de Coulanges: "Cité et ville n'étaient pas des mots synonymes chez les anciens $[. . .]^{5}$.» Ainsi, il faut distinguer la cité, en tant que communauté humaine, soit une "association religieuse et politique ", et la ville qui est, en quelque sorte, son corps physique, le lieu de réunion, de domicile, le « sanctuaire » de cette association, à la fois matérielle et symbolique. Par conséquent, la communauté précède la trame urbaine, dont elle est le produit.

Dans l'Antiquité, l'interrogation sur ces deux phénomènes parallèles, la formation d'une communauté politiquement organisée (la cité), d'une part, et l'urbanisation (la ville), d'autre part, rencontre un point de convergence dans la pensée philosophique sur la cité idéale ${ }^{6}$. Les deux figures centrales pour cette question, Platon et Aristote, ont, tous les deux, mais chacun à sa façon, réfléchi à l'interaction entre l'aménagement des espaces, urbains et ruraux, et l'organisation politique la meilleure ${ }^{7}$.

Dans l'historiographie contemporaine, depuis l'ouvrage fondateur de Numa Fustel de Coulanges, la cité antique a fait l'objet de nombreuses approches ${ }^{8}$, d'une manière constante et sous des angles sans cesse renouvelés 9 . Il est bien entendu impossible, dans l'espace restreint de notre contribution, de reprendre en détail ce parcours historiographique qui continue à donner lieu à une abondante bibliographie ${ }^{10}$. Il ne s'agit donc pas ici de se livrer à une synthèse des travaux existants.

5 Dans la perspective qui a animé les ateliers dont ce dossier est issu, on rappellera néanmoins les travaux fondateurs de Max Weber ${ }^{11}$ et de Gustave Glotz ${ }^{12}$. Le premier envisageait une fourchette chronologique très large, de l'Antiquité aux temps modernes, alors que le second se limitait, quant à lui, à la seule cité grecque antique. 
Dans la diversité de leurs approches et de leurs enjeux, leurs réflexions ont contribué à mettre en exergue la difficulté qu'on peut éprouver à définir la cité antique au cours de son histoire. L'œuvre de Moses I. Finley est également essentielle, et les réflexions qu'elle propose sur l'idée de "cité antique ", tant grecque que romaine, ont donné lieu à des relectures critiques très pertinentes ${ }^{13}$. On doit à Victor Ehrenberg d'avoir posé clairement, et pour la première fois, la question de l'émergence de la cité ${ }^{14}$. Dans l'enquête sur la poléogenèse, François de Polignac avait d'emblée souligné que le recours à la notion de polis posait un problème majeur: lorsqu'elle est utilisée par l'historien, son arrière-plan institutionnel peut en effet fausser l'analyse dans la mesure où elle mobilise des modes de pensée et des outils conceptuels qui reflètent davantage les débats de l'historiographie moderne que les problèmes historiques du monde antique ${ }^{15}$.

6 Le cas de la cité grecque des époques archaïque et classique a été envisagé par le Copenhagen Polis Center, sous la direction de Mogens H. Hansen, dans une entreprise de longue haleine, qui a débouché sur de nombreuses publications en proposant des comparaisons entre des modèles culturellement divers ${ }^{16}$. Cette démarche a contribué à mettre en évidence le caractère polysémique ou plutôt variable de la définition même de cité, en soulignant comment le regard que l'on porte sur elle en tant qu'objet historique est forcément orienté par les mots qui la qualifient.

7 Pour notre propre perspective, visant à confronter la notion de cité avec les formes urbaines qui peuvent y être associées ${ }^{17}$, le volume La Città greca antica, dirigé par Emanuele Greco, constitue assurément un jalon essentiel ${ }^{18}$. Méthodologiquement, nous en partageons le positionnement, en envisageant à la fois l'archéologie et l'histoire, dans une approche dialectique qui exclut tout raccourci combinatoire ${ }^{19}$. De fait, si l'éclairage institutionnel, perspective traditionnelle depuis Fustel de Coulanges, ne permet pas toujours de cerner dans sa spécificité la polis notamment à l'époque archaïque $^{20}$, ce projet propose de la rechercher dans les multiples facettes qu'elle génère et qui, au bout du compte, l'engendrent. Une réflexion sur la cité appréhendée au-delà de l'approche dichotomique, fondée sur sa nature physique et politique, est aujourd'hui possible grâce notamment à l'acquisition de nouvelles données en archéologie, architecture et urbanisme ${ }^{21}$. L'archéologie a d'emblée constitué la voie d'accès privilégiée pour saisir notre objet dans sa dimension matérielle, pour comprendre comment l'espace urbain a été façonné par les sociétés et comment il a déterminé certaines de leurs spécificités.

8 Avant d'aller plus avant, il peut s'avérer utile d'approfondir les raisons de la recherche d'une définition. Notre démarche part d'un constat: si les mots latins ou grecs demeurent, les réalités administratives et sociales qu'ils désignent peuvent en revanche varier $^{22}$. Nous devrions dès lors davantage suivre la mise en garde de Marc Bloch: l'historien contemporain utilise souvent la terminologie employée dans les sources anciennes, en dépit de la distance chronologique, des contingences historiques et des éventuels glissements sémantiques. Or, il faut situer toute analyse du vocabulaire dans le temps ${ }^{23}$. François Favory l'a bien souligné : il ne s'agit pas d'interdire l'usage des vocables anciens, mais de demander «que l'on respecte leur charge sémantique et leur contexte d'origine et qu'on ne substitue pas l'affirmation à l'hypothèse ${ }^{24}$ ». Comme l'a précisé Edmond Lévy,

Les modernes savent - ou croient savoir - mieux que les Anciens ce qu'est une cité, ce qui leur permet de reprocher à Hérodote d'appeler indûment telle localité une polis, d'affirmer que telle polis n'est pas une vraie polis ou de traduire à 
l'occasion, quand le texte grec ne correspond pas aux conceptions modernes, polis par « petite cité », « bourgade » ou « établissement $»^{25}$. percevaient la ville, et aucune théorie antique sur la ville ou l'aménagement urbain ne nous est parvenue complète. Le terme polis relève par ailleurs d'une certaine ambivalence dans la mesure où il recouvre à la fois une réalité politique et topographique qui est elle-même ambiguë, le mot pouvant recouvrir l'ensemble du territoire sur lequel s'exerce l'autorité de l'État - avec un centre urbain et une campagne -, ou le seul centre urbain ${ }^{26}$. Dans son ouvrage, Luigi Maria Caliò s'interroge justement sur cette polysémie. Nommer la polis, la décrire, la restituer, en confrontant textes et vestiges, revient d'abord à tenter de cerner la relation entre ces deux notions que sont la cité et la ville, entre les hommes et leurs espaces, entre l'individu et la collectivité :

Polis in realtà è una parola di difficile traduzione che nasce probabilmente per indicare un agglomerato fisico, ma che perde in età post-micenea tale accezione per indicare una comunità che tra l'età del ferro e l'Atene periclea si forma tra contraddizioni irrisolte e resistenze gentilizie ${ }^{27}$.

10 Parvenir à déterminer ce qu'ont été les rapports des sociétés de l'Antiquité classique avec la cité et la ville, et appréhender s'ils ont été les mêmes dans le monde grec et dans le monde romain, est une des voies par lesquelles nous pouvons apprendre à mieux connaître ces sociétés. D'un point de vue historique, il nous faut donc encadrer d'abord la différence entre polis et ciuitas. Le latin distingue clairement ciuitas d'urbs, mais la ville doit être comprise à la fois comme urbs (l'espace où les bâtiments sont installés) et ciuitas, c'est-à-dire comme un espace bâti, comme une association sociale de citoyens et comme une communauté politique ${ }^{28}$. Edmond Lévy a ainsi remarqué que le même mot polis peut avoir été utilisé par un même auteur ancien pour désigner trois réalités différentes: la ville et sa campagne (urbs), la communauté installée dans cette ville (ciuitas), et enfin l'organisation politique de cette communauté (res publica) ${ }^{29}$. Ciuitas est pour cela utilisé comme synonyme d'urbs et comme traduction de polis. Mais «[...] in Grecia il 'cittadino' nasce dalla 'città', per la città e nella città, mentre a Roma vale il contrario, è dal cittadino e per il cittadino che nasce la città [... $]^{30} »$. Dès lors, les termes de polis et ciuitas relèvent de conceptions différentes chez les Grecs et chez les Romains. Il s'agit alors de recenser, analyser et comparer le vocabulaire utilisé pour définir et décrire la ville et la cité, de questionner la traduction des termes grecs et latins dans nos langues contemporaines.

11 Dans plusieurs cas, comme on pourra le voir précisément dans les contributions rassemblées ici ${ }^{31}$, un même terme peut, dans les sources antiques, désigner des réalités différentes. Inversement, des réalités semblables peuvent être nommées par des mots distincts. D'entrée de jeu, il faut tenir compte de la diversité des situations et adopter une distance critique dans l'analyse des sources et dans le choix pertinent des concepts, grâce à la parole de spécialistes de différents domaines et époques. Ces vocables peuvent, d'autre part, recouvrir, pour un archéologue et un historien, des réalités fort différentes ${ }^{32}$. Des questions jaillissent alors, qui reposent à la fois sur le vocabulaire et sur nos pratiques.

12 La polis est une construction mentale : les premières descriptions de villes grecques ou romaines sont liées à des images géométriques et symboliques qui les relient intimement à l'aspect social et communautaire de leur constitution. Une telle démarche nous invite à examiner les idées et les « images » mobilisées pour concevoir 
la ville ${ }^{33}$. La polysémie du terme latin forma, qui désigne aussi bien la «forme», le «tableau général» que le "plan ${ }^{34}$, en est un indice. La polis grecque était parfois figurée comme une série de cercles concentriques sur le bouclier d'Achille (Homère, Iliade, XVIII, 478-608) 35 , ou la ville romaine comme la Roma quadrata («Rome carrée »), idéalement tracée suivant les prescriptions rituelles par Romulus sur le Palatin ${ }^{36}$. Le parallélisme entre Rome et les cités grecques, Athènes notamment, semble avoir été même revendiqué par les élites romaines républicaines peut-être avant Fabius Pictor $^{37}$.

13 L'idée que la ville antique dispose d'une forme particulière, un plan urbain, demeure jusqu'à nos jours, même si la réalité des agglomérations devait être beaucoup plus irrégulière ${ }^{38}$, échappant ainsi à toute classification trop stricte. On observe dans l'historiographie contemporaine - et dans des langues et traditions archéologiques diverses - le recours à des catégories descriptives plus variées ${ }^{39}$ : habitat, pour désigner la maison; habitat groupé, comunità di villaggio, small towns, Kleinstädte, agglomération secondaire, bourg, bourgade, village, centro protourbano, ou, de façon plus vague, site, établissement ou settlement, pour désigner les différents types d'agglomérations ${ }^{40}$.

14 L'attention portée à l'examen du vocabulaire mobilisé pour nommer ces deux réalités, la cité et la ville, devrait permettre de mettre en lumière, par-delà l'écume du particulier, les éléments essentiels qui, en premier lieu, faisaient qu'une entité pouvait être perçue comme une cité et, deuxièmement, constituaient l'identité spécifique d'une cité donnée, aussi bien dans sa définition en tant que communauté de citoyens, que dans son implantation urbaine ${ }^{41}$.

15 Comprendre les processus de formation et d'évolution des cités dans les mondes antiques implique nécessairement l'analyse des relations de tension, voire d'antagonisme, à différentes époques et dans des contextes géographiques distincts, entre un centre urbanisé (la ville) et le territoire ${ }^{42}$. L'observation de l'aménagement urbain et des solutions architecturales adoptées éclaire leur interrelation avec la nature, le rôle et les modes d'exploitation du territoire environnant. Une enquête sur la ville antique limitée au seul habitat nous donnerait à voir une image partielle et partiale, pour ne pas dire biaisée: un de nos objectifs est au contraire de montrer comment, dès le début, les villes apparaissent en tant qu'organismes territoriaux complexes $^{43}$, sans perdre de vue le milieu autochtone ${ }^{44}$ qui représente un point d'observation de première importance ${ }^{45}$.

16 À cet égard, une compréhension exacte du vocabulaire utilisé dans la description de l'espace est également importante ${ }^{46}$ : outre «polis ${ }^{47}$ ", il y a de nombreux termes qui manquent d'un sens précis ${ }^{48}$, en premier lieu celui de "chôra». Ce mot peut être compris précisément dans le double sens de «territoire entourant la ville » et de zone de pouvoir qui peut dépasser les limites strictement prévues pour le contrôle civique ${ }^{49}$. Par ailleurs, même si traditionnellement la recherche s'intéresse principalement aux vestiges construits, traces tangibles d'un paysage urbain disparu, il ne faut pas oublier que la forme urbaine est le résultat d'une articulation entre espaces construits et espaces vides: rues, places, mais aussi terrains sans fonction particulière ${ }^{50}$. Ce renouveau théorique est également lié à une prise de conscience de l'importance du proasteion, du périurbain. En effet, dès le viI siècle av. J.-C., ce terme montre que l'extra-urbain est "conceptualisé » et qu'il correspond sûrement à une composante précise dans l'appréhension par les Grecs de l'espace habité ${ }^{51}$. Julien du Bouchet a mis en évidence les évolutions du sens de ce mot, notamment à l'époque impériale, où, sous 
l'influence des manières latines de désigner les domaines suburbains (suburbanum), proasteion désigne alors des propriétés situées hors de la ville, précisément dans cet espace devant la ville, mais avant la campagne ${ }^{52}$.

17 Le mot polis et certains de ses dérivés posent en outre la question du regard porté sur les villes du monde gréco-romain et celles des autres. Si les interrogations sur la cité grecque, notamment au moment de sa formation, demeurent au centre de nos préoccupations $\mathrm{s}^{53}$, le recours à une démarche comparatiste, prenant en compte les différents phénomènes citadins des cités étrusques, romaines impériales, gauloises ou gallo-romaines dans une fourchette chronologique large, de l'archaïsme jusqu'à l'Antiquité tardive, a permis de réfléchir, lors de nos rencontres, à la fois sur les variations d'échelles et sur la diversité des situations locales. Il s'agissait par ce biais de s'interroger sur les discours et le vocabulaire relatifs aux villes et cités antiques, et sur les représentations qu'ils véhiculent.

Ainsi, un aspect essentiel de ce dossier concerne la formation des agglomérations italiques, étrusques et gauloises. Des fouilles récentes et de nombreuses études sont venues renouveler cette question, en mettant au jour des établissements et des agglomérations relevant d'une expérience « urbaine » pour des périodes ou des régions pour lesquelles l'idée même d'«urbanisation" n'était pas acquise dans l'historiographie traditionnell ${ }^{54}$. Par exemple, on retenait généralement que la majeure partie de l'hinterland indigène de la péninsule italienne était restée à l'écart de l'« Italie des cités ». Elle n'aurait accédé que tardivement, voire de manière limitée, au fait urbain. Ce modèle est aujourd'hui largement remis en question par les recherches sur la Lucanie notamment :

Pour pouvoir parler un jour de "villes » italiques, il faut cependant davantage qu'un changement de paradigme. Nous devons aussi modifier notre manière même de mener l'enquête, l'échelle et la focale de nos investigations. À partir des mêmes sondages limités, on le sait bien, peuvent être affirmés une chose et son contraire. Ce qui nous manque encore, c'est la connaissance extensive, non extrapolée, des trames construites ${ }^{55}$.

19 Ces dernières décennies, l'archéologie a beaucoup contribué à faire progresser notre connaissance des origines de l'urbanisme antique ${ }^{56}$, en suscitant sans cesse de nouvelles interrogations et en ouvrant la voie à de nouvelles approches ${ }^{57}$. Cela nous mène à une question plus large. Comme l'explique Stéphane Bourdin ${ }^{58}$, dans le bassin occidental de la Méditerranée, c'est à partir du début de l'âge du fer que les archéologues entrevoient la mise en place d'agglomérations urbaines, en même temps que se structurent les pouvoirs politiques (cités, royaumes). Si les auteurs antiques qualifiaient de «ville » (polis, urbs, oppidum) toutes les agglomérations qui étaient le siège d'une communauté politique autonome, indépendamment de la réalité matérielle, les archéologues ont longtemps refusé d'employer ce vocabulaire pour des sites, comme les oppida celtiques, qui ne correspondaient pas au schéma classique de la ville antique ${ }^{59}$, préférant des termes comme " proto-urbain ». Actuellement, la tendance est plutôt à interpréter les agglomérations protohistoriques d'Europe continentale comme les manifestations d'une véritable expérience urbaine ${ }^{60}$. La documentation se révèle defait très hétérogène ${ }^{61}$.

Dans les sociétés gauloises, celtiques et ibériques, la manifestation archéologique de l'oppidum est autant polymorphe que le terme est polysémique ${ }^{62}$. Les travaux archéologiques menés en France depuis trente ans, à partir notamment de la publication de l'atlas des agglomérations de la Franche-Comté puis de la Bourgogne ${ }^{63}$, 
ont permis de mettre au jour de nombreuses agglomérations dans les territoires des cités gallo-romaines. Les nouvelles données acquises grâce au développement de l'archéologie programmée et préventive ont remis en cause l'idée que le phénomène urbain antique serait limité aux seuls chefs-lieux ${ }^{64}$.

L'oppidum est, pour la société gauloise, le cœur de l'organisation politique, économique et territoriale, comme l'indiquent notamment les fouilles de la capitale des Éduens, bien que le phénomène soit de courte durée ${ }^{65}$ : Bibracte est, à l'instar de nombreux autres oppida, abandonné après la conquête romaine, aux alentours du changement d'ère, au bénéfice d'une ville neuve, Augustodunum (Autun). Quant à savoir où se trouvaient les Éduens dans l'intervalle entre les dernières traces d'occupation de Bibracte, situées vers 15 av. J.-C., et la création d'Autun, William Van Andringa rappelle, à juste titre, qu'entre le choix de création d'une nouvelle ville et son aménagement, voire sa monumentalisation, il y a des temps différents avec d'importantes réalisations, des chantiers de construction et autres travaux de terrassement ${ }^{66}$.

$\mathrm{Si}$, jusqu'à nos jours, l'analyse du processus de création urbaine dans les provinces nouvellement conquises s'est essentiellement focalisée sur le modèle classique de la ville méditerranéenne et sur l'idée d'un Idealtyp importé d'Italie, l'enquête archéologique montre, quant à elle, la mise au jour, dans de nombreux cas, d'une occupation gauloise pouvant parfois s'apparenter à une ville ${ }^{67}$. Un des acquis les plus importants des recherches récentes est que l'émergence des nouveaux chefs-lieux n'est pas le produit d'un phénomène univoque, et qu'il est bien plus en continuité avec l'époque protohistorique qu'on ne l'a longtemps $\mathrm{cru}^{68}$.

23 En Gaule chevelue, par exemple, plusieurs facteurs tiennent un rôle déterminant dans le processus de création urbaine : l'héritage protohistorique donc, dont le poids doit sans doute être réévalué fortement par rapport à la perception traditionnelle; la dynamique propre au développement urbain avant la conquête; la place des grands sanctuaires laténiens ; l'implantation du nouveau réseau viaire, qui a une incidence sur les équilibres géographiques et économiques antérieurs; l'implication des acteurs locaux, les élites, dont le degré d'insertion dans les nouveaux cadres politiques romains et la richesse conditionnent leur capacité d'évergétisme ${ }^{69}$.

Des différences existent indéniablement entre le monde classique, gréco-romain, et d'autres populations, italiques, celtiques, gauloises: la ville et la cité antiques sont apparues sous des formes multiples qui ne permettent pas une définition unique ${ }^{70}$. Il ne saurait donc être question de chercher à mettre en évidence un modèle unique, ce qui s'apparenterait à une sorte d'aporie, voire de "non-sens", mais plutôt de tenter d'appréhender, dans toute leur diversité géographique, culturelle et chronologique, les moyens et les étapes d'une genèse parfois longue et discontinue. C'est précisément par l'approche comparatiste, avec la nécessaire prise en considération du contexte historique de l'apparition et de l'usage des mots - reflet de systèmes de représentation collectifs - et la mise en perspective des données archéologiques récentes, que l'on peut identifier les nœuds théoriques essentiels et spécifier les problèmes les plus importants. Il s'en dégage ainsi un éclairage nouveau. La circulation des termes et leur diversité renseignent sur la difficulté à rendre compte d'une réalité spatiale archéologiquement diversifiée que l'on souhaite catégoriser. Or, étant par définition polyédrique, le phénomène urbain antique doit être abordé au prisme de ses différentes manifestations historiques, sous différents angles, en faisant appel à des sources et documents divers, en considérant des paramètres variés, en accordant une attention 
particulière au débat sur les concepts et leur usage, sans nier l'importance des peuples indigènes et leur autonomie historique.

Les divers participants à ce dossier ont des points de départ distincts, les concepts ne sont pas plus identiques que les méthodes de recherche mobilisées et les traditions historiques revendiquées; les contextes institutionnels et disciplinaires divergent également. Si des modèles communs agissent comme des outils, les configurations étudiées apparaissent toutefois diverses. Certes, s'intéresser au phénomène urbain dans plusieurs contextes, en Grèce, en Italie, en Gaule, en péninsule Ibérique, en Afrique, suppose que l'on adopte d'entrée de jeu le prisme de l'interculturalité ${ }^{71}$. Cette dernière reconnaît l'altérité et la variété, tout en soulignant le caractère dynamique de chacune des cultures en contact; elle admet également des interactions et des emprunts, ainsi que la réciprocité. En effet, «l'invention et la reproduction de la cité, entre valeurs et négociations, est aussi un phénomène de transmission culturelle ${ }^{72} »$. Cette affirmation ne doit obscurcir pour autant le fait que le phénomène urbain ait, dans de nombreuses civilisations, des racines endogènes ${ }^{73}$, répondant ainsi également à une préoccupation de « décolonisation » de l'historiographie contemporaine ${ }^{74}$.

La prise en compte du phénomène urbain apparaît donc comme une gageure qu'il est difficile de relever sans opérer avec prudence et transversalité. Celle-ci oblige à solliciter de nombreuses compétences, réparties dans des champs disciplinaires distincts. Il s'agit justement ici de parcourir ces domaines de manière transversale, au sein d'un cadre conceptuel renouvelé que les approches historiques et les pratiques archéologiques contemporaines permettent d'explorer.

\section{BIBLIOGRAPHIE}

ANDREAU Jean, HARTOG François et al., La Cité antique ? À partir de l'œuvre de M. I. Finley, Opus. Rivista internazionale per la storia economica e sociale dell'Antichità, 6-8, Rome, Opus, 1987-1989.

ATTEMA Peter A. J., SEUBERS Jorn F. \& WILLEMSEN Sarah Lea (éd.), Early States, Territories and Settlements in Protohistoric Central Italy (Proceedings of a specialist conference at the Groningen Institute of Archaeology of the University of Groningen, 2013), Groningue, University of Groningen, Groningen Institute of Archaeology, 2016.

BARRAL Philippe, COQUET Nicolas \& NOUVEL Pierre, « Les agglomérations antiques de FrancheComté : bilan et perspectives ", dans J.-F. Piningre \& A. Richard (éd.), Dix ans d'archéologie en Franche-Comté (1995-2005), Bilan scientifique (hors-série), SRA de Franche-Comté, 2012, p. 149-169. Disponible en ligne sur <halshs-00772130>.

BELARTE Maria Carme \& PLANA-MALLART Rosa (éd.), El paisatge periurbà a la Mediterrània occidental durant la protohistòria i l'antiguitat [Le paysage périurbain en Méditerranée occidentale pendant la Protohistoire et l'Antiquité], Tarragone, Institut Català d'Arqueologia Clàssica, 2012.

BELARTE Maria Carme, NOGUERA Jaume, PLANA-MALLART Rosa \& SANMARTí Joan (éd.), Urbanization in Iberia and Mediterranean Gaul in the First Millennium BC, Tarragone, Institut Català d'Arqueologia Clàssica, coll. « Trama, 7 », à paraître. 
BERNAL Martin, Black Athena: The Afroasiatic Roots of Classical Civilization, vol. 1 : The Fabrication of Ancient Greece 1785-1985; vol. 2 : The Archaeological and Documentary Evidence ; vol. 3 : The Linguistic Evidence, New Brunswick (NJ), Rutgers University Press, 1987 (vol. 1), 1991 (vol. 2), 2006 (vol. 3).

BERNAL Martin, Black Athena Writes Back: Martin Bernal Responds to His Critics, Londres, Duke University Press, 2001.

BERTRAND Jean-Marie, « L'Utopie magnète : réflexions sur les Lois de Platon », dans M. H. Hansen (éd.), The Imaginary Polis (Acts of the Copenhagen Polis Centre, 7), Copenhague, Copenhagen Polis Center, 2005, p. 152-163.

BLOCH Marc, Apologie pour l'histoire ou Métier d'historien, Paris, Armand Colin, 2013 (1949).

BOUCHET Julien du, « Expériences et représentations de l'espace en grec ancien », dans

P. Guisard \& C. Laizé (éd.), Expériences et représentations de l'espace, Paris, Ellipses, 2012, p. 9-21.

BOURDIN Stéphane, « Pré-urbain, proto-urbain, urbain : les agglomérations et les archéologues », dans S. Bourdin, M. Paoli \& A. Reltgen-Tallon (éd.), La Forme de la ville : de l'Antiquité à la Renaissance, Rennes, Presses universitaires de Rennes, 2015, p. 353-363.

BOURDIN Stéphane, PAOLI Michel \& RELTGEN-TALLON Anne, « Introduction », dans S. Bourdin, M. Paoli \& A. Reltgen-Tallon (éd.), La Forme de la ville : de l'Antiquité à la Renaissance, Rennes, Presses universitaires de Rennes, 2015, p. 9-19.

BRIQUEL Dominique, « Rome comme ville étrusque », dans P. Fleury \& O. Desbordes (éd.), Roma illustrata : représentations de la ville (Actes du colloque international de Caen, 6-8 octobre 2005), Caen, Presses universitaires de Caen, 2008, p. 63-84.

BRUHNS Hinnerk, « La Cité antique de Max Weber », dans La Cité antique ? À partir de l'œuvre de M. I. Finley, Opus. Rivista internazionale per la storia economica e sociale dell'antichità, 6-8, 1987-1989, p. 29-42.

BRUHNS Hinnerk \& NIPPEL Wilfried, « Max Weber, M. I. Finley et le concept de cité antique », dans La Cité antique ? À partir de l'œuvre de M. I. Finley, Opus. Rivista internazionale per la storia economica e sociale dell'antichità, 6-8, 1987-1989, p. 27-28.

BRUN Patrice \& CHAUME Bruno, « Une éphémère tentative d'urbanisation en Europe centreoccidentale durant les $\mathrm{VI}^{\mathrm{e}}$ et $\mathrm{V}^{\mathrm{e}}$ siècles av. J.-C. ? ", Bulletin de la Société préhistorique française, 110 (2), 2013, p. 319-349. Disponible en ligne sur <https://doi.org/10.3406/bspf.2013.14263>.

CARANDINI Andrea, Remo e Romolo: dai rioni dei Quiriti alla città dei Romani (775/750-700/675 a.C. circa), Turin, Einaudi, 2006.

CARSANA Chiara \& SCHETTINO Maria Teresa (éd.), Utopia e utopie nel pensiero storico antico, Rome, L'Erma di Bretschneider, 2008.

CARSANA Chiara \& ZIZZA Cesare, « “Dónde fundaremos nuestra ciudad?” Lugares y constituciones ideales en Platón, Aristóteles y Cicerón », DHA, 45 (2), 2019, p. 167-196.

CALIÒ Luigi Maria, Asty: studi sulla città greca, Rome, Quasar, 2012.

CALIÒ Luigi Maria, « La città immaginata. Raffigurazione e realtà urbana nella Grecia classica », Thiasos. Rivista di archeologia e architettura antica, 5 (2), 2016, p. 33-47.

CALIÒ Luigi Maria, « Dalla polis immaginata all'asty delle immagini. Percorsi di analisi dell'immagine di città nel mondo antico ", dans M. Livadiotti, R. Belli Pasqua, L. M. Caliò \& G. Martines (éd.), Theatroeideis: l'immagine della città, la città delle immagini (Atti del Convegno internazionale, Bari, 15-19 giugno 2016), Thiasos Monografie 11, Rome, Quasar, 2018, p. 15-45. 
CASEVITZ Michel, «Remarques sur l'histoire de quelques mots exprimant l'espace en grec », REA, 100, 1998, p. 417-435.

CAZANOVE Olivier de \& BOURDIN Stéphane, «Ignobilia oppida Lucanorum : recherches sur les "villes sans renom" des Lucaniens de l'intérieur (Serra del Cedro, Civita di Tricarico, sites d'habitat et sanctuaires avoisinants) ", dans O. de Cazanove \& A. Duplouy (éd.), La Lucanie entre deux mers : archéologie et patrimoine (Actes du colloque international, Paris, 5-7 novembre 2015), Naples, Centre Jean Bérard, 2019, p. 311-331.

COARELLI Filippo, Palatium. Il Palatino dalle origini all'impero, Rome, Quasar, 2012.

CORBIER Mireille, « Les mots de la ville et de la cité dans l'Empire romain », Topoi. Orient-Occident, suppl. 12, 2013, p. 517-542.

COUDRY Marianne \& SCHETTINO Maria Teresa (éd.), L'utopie politique et la cité idéale, Politica antica, Rivista di prassi e cultura politica nel mondo greco e romano, Bari, Edipuglia, 2015.

CRIFÒ Giuliano, Civis: la cittadinanza tra antico e moderno, Rome/Bari, Laterza, 2004.

DARCOS Xavier, HARTOG François \& MANENT Pierre (éd.), Relire Fustel de Coulanges : la cité antique cent cinquante ans après (1864-2014), Bulletin de l'Académie des sciences morales et politiques, 8, Paris, Académie des sciences morales et politiques, 2015.

DAVIES John K., « Il discorso sulla polis Greca oggi », dans Poleis e politeiai nella Magna Grecia arcaica e classica (Atti del convegno di studi sulla Magna Grecia LIII), Tarente, Istituto per la Storia e l'Archeologia della Magna Grecia, 2016, p. 7-28.

DUPLOUY Alain, Construire la cité : essai de sociologie historique sur les communautés de l'archaïsme grec, Paris, Les Belles Lettres, 2019.

EHRENBERG Victor, « When Did the Polis Rise? », Journal of Hellenic Studies, 57 (2), 1937, p. 147-159.

ELDEN Stuart, « Genealogy, Ontology and the Political: Three Conceptual Questions to Engin Isin », Political Geography, 24 (3), 2005, p. 355-359.

ÉTIENNE Roland, « Compte rendu de R. Osborne, B. Cunliffe (éd.), Mediterranean Urbanisation 800600 BC, Oxford (2005)», Topoi, 15 (2), 2007, p. 591-596.

ÉTIENNE Roland, «La notion de proasteion dans les textes grecs », dans P. Darcque, R. Étienne et al. (éd.), Proasteion : recherches sur le périurbain dans le monde grec, Paris, 2013, p. 13-27.

FANTASIA Ugo, « Platone e Aristotele sull'organizzazione della chora », Annali della Scuola Normale Superiore di Pisa, 3, 1975, p. 1255-1274.

FAVORY François, «La dure condition des agglomérations secondaires », Les Nouvelles de l'archéologie, 127, 2012, p. 40-44.

FERNÁNDEZ-GÖTZ Manuel, « Urbanization in Iron Age Europe: Trajectories, Patterns, and Social Dynamics ", Journal of Archaeological Research, 26, 2018, p. 117-162. Disponible en ligne sur <https://doi.org/10.1007/s10814-017-9107-1>.

FERNÁNDEZ-GÖTZ Manuel \& KRAUSSE Dirk (éd.), Eurasia at the Dawn of History: Urbanization and Social Change, Cambridge, Cambridge University Press, 2016.

FICHTL Stephan, La ville celtique : les oppida de 150 av. J.-C. à 15 ap. J.-C., Paris, Errance, 2005 (2000).

FICHTL Stephan, « Les agglomérations gauloises de la fin de l'âge du fer en Europe celtique (III ${ }^{\mathrm{e}} \mathrm{I}^{\mathrm{er}}$ siècle av. J.-C.) », dans D. Garcia (éd.), L'habitat en Europe celtique et en Méditerranée préclassique : domaines urbains, Arles, Errance, 2013, p. 19-43. 
FINLEY Moses I., « The Ancient City: From Fustel de Coulanges to Max Weber and Beyond », dans B. D. Shaw \& R. P. Saller (éd.), Economy and Society in Ancient Greece, Londres, Chatto and Windus, 1981, p. 3-23.

FINLEY Moses I., « Max Weber and the Greek City-State », dans M. I. Finley (éd.), Ancient History: Evidence and Models, New York, Viking, 1985, p. 88-103.

FINOCCHIETTI Luigi \& LUBTCHANSKY Natacha (éd.), Aux pieds des murs dans l'Italie préromaine. Pour une définition du périurbain dans les cités italiques et italiotes, Naples, Centre Jean Bérard, à paraître.

FRÖHLICH Pierre, «L'inventaire du monde des cités grecques. Une somme, une méthode et une conception de l'histoire », Revue historique, 655, 2010, p. 637-676.

FULMINANTE Francesca, The Urbanization of Rome and Latium Vetus: From the Bronze Age to the Archaic Era, Cambridge, Cambridge University Press, 2014.

FUSTEL DE COULANGES Numa, La Cité antique, Paris, Flammarion, 1984 (1864).

GARCIA Dominique (éd.), L'habitat en Europe celtique et en Méditerranée préclassique : domaines urbains, Arles, Errance, 2013.

GARCIA Dominique, « La ville préromaine en Gaule méditerranéenne », dans Villes méditerranéennes, civilisation et développement (Actes de la conférence internationale), Barcelone, Union académique internationale/IEC, 2015, p. 66-74.

GAWANTKA Wilfried, Die Sogenannte Polis: Entstehung, Geschichte und Kritik der modernen althistorischen Grundbegriffe der griechische Staat, die griechische Staatsidee, die Polis, Stuttgart, Steiner, 1985.

GIANGIULIO Maurizio, «Stato e statualità nella polis: riflessioni storiografiche e metodologiche. Ovvero del buon uso di Max Weber e del paradigma dello stato moderno », dans S. Cataldi (éd.), Poleis e politeiai: esperienze politiche, tradizioni letterarie, progetti costituzionali, Alessandria, Edizioni dell'Orso, 2004, p. 31-53.

GINZBURG Carlo, « Our Words, and Theirs: A Reflection on the Historian's Craft, Today », dans S. Fellman \& M. Rahikainen (éd.), Historical Knowledge: In Quest of Theory, Method and Evidence, Newcastle upon Tyne, Cambridge Scholars Publishing, 2012, p. 97-119.

GRANDAZZI Alexandre, « La Roma quadrata : mythe ou réalité ? », MEFRA, 105 (2), 1993, p. 493-545. GRECO Emanuele (éd.), La Città greca antica: istituzioni, società e forme urbane, Rome, Donzelli editore, 1999.

GRECO Emanuele (éd.), Teseo e Romolo: le origini di Atene e Roma a confronto (Atti del convegno internazionale di studi), Athènes, Scuola archeologica italiana di Atene, 2005.

GRECO Emanuele, « L'archeologia della polis in Magna Grecia », dans Poleis e politeiai nella Magna Grecia arcaica e classica (Atti del convegno di studi sulla Magna Grecia LIII), Tarente, Istituto per la Storia e l'Archeologia della Magna Grecia, 2016, p. 65-90.

GRECO Emanuele, Ippodamo di Mileto: immaginario sociale e pianificazione urbana nella Grecia classica, Paestum, Pandemos, 2018.

GROS Pierre, «Villes et "non-villes" : les ambiguïtés de la hiérarchie juridique et de l'aménagement urbain », dans P. Gros (éd.), Villes et campagnes en Gaule romaine (Actes du $120^{\mathrm{e}}$ Congrès national des sociétés historiques et scientifiques), Paris, CTHS, 1998, p. 11-25. 
HANOUNE Roger (éd.), Les villes romaines du nord de la Gaule : vingt ans de recherches nouvelles (Actes du XXV e Colloque international de HALMA-IPEL UMR CNRS 8164), Lille, Revue du Nord, 2007.

HANSEN Mogens H. «Introduction. The Polis as Citizen-State », dans M. H. Hansen (éd.), The Ancient Greek City-State (Symposium on the occasion of the 250th anniversary of the Royal Danish academy of sciences and letters, 1-4 July 1992), Copenhague, Munksgaard, 1993, p. 7-29.

HANSEN Mogens H., «Polis as the Generic Term for State », dans T. H. Nielsen (éd.), Yet More Studies in the Ancient Greek "Polis", Stuttgart, Franz Steiner Verlag, 1997a, p. 9-15.

HANSEN Mogens H. (éd.), The Polis As an Urban Centre and As a Political Community (Symposium, 2931 August 1996), Copenhague, Det Kongelige Danske Videnskabernes Selskab, 1997b.

HANSEN Mogens H., Polis et cité-État. Un concept antique et son équivalent moderne, trad. fr., Paris, Les Belles Lettres, 2001.

HANSEN Mogens H. \& NIELSEN Thomas H. (éd.), An Inventory of Archaic and Classical Poleis: An Investigation Conducted by The Copenhagen Polis Centre for the Danish National Research Foundation, Oxford, Oxford University Press, 2004.

HÖLKESKAMP Karl-Joachim, « "Ptolis" and "agore". Homer and the Archaeology of the CityState ", dans F. Montanari \& P. Ascheri (éd.), Omero tremila anni dopo (Atti del congresso di Genova, 6-8 luglio 2000), Rome, Edizioni di storia e letteratura, 2002, p. 297-342.

HÖLSCHER Tonio, « Lo spazio pubblico e la formazione della città antica », dans E. Greco (éd.), Teseo e Romolo: le origini di Atene e Roma a confronto (Atti del convegno internazionale di studi), Athènes, Scuola archeologica italiana di Atene, 2005, p. 211-238.

HUMM Michel, « Rome, une "cité grecque” prise par les Hyperboréens », Ktèma, 42, 2017, p. 53-72. HUOT Jean-Louis (éd.), La ville neuve, une idée de l'Antiquité ?, Paris, Errance, 1988.

LARGUINAT-TURBATTE Gabrièle, « Les espaces vides dans les villes grecques. Ionie et Carie principalement, $\mathrm{IV}^{\mathrm{e}}-\mathrm{I}^{\mathrm{er}}$ S. a.C. », Histoire urbaine, 52, 2018, p. 121-136.

LEFKOWITZ Mary R. \& MACLEAN ROGERS Guy (éd.), Black Athena Revisited, Chapel Hill, University of North Carolina Press, 1996.

LEPORE Ettore, «Per una fenomelogia storica del rapporto città-territorio in Magna Grecia ", dans La città e il suo territorio (Atti del convegno di studi sulla Magna Grecia VII), Tarente, Istituto per la Storia e l'Archeologia della Magna Grecia, 1967, p. 29-66.

LEPORE Ettore, « Problemi dell'organizzazione della chora coloniale », dans M. I. Finley (éd.), Problèmes de la terre en Grèce Ancienne, Paris, Mouton \& Co., 1973, p. 15-47.

LEVEAU Philippe, « Du bon usage de l'anachronisme dans la recherche archéologique sur les agglomérations protohistoriques et gallo-romaines ", dans D. Garcia (éd.), L'habitat en Europe celtique et en Méditerranée préclassique : domaines urbains, Arles, Errance, 2013, p. 9-18.

LÉVY Edmond, « La cité grecque : invention moderne ou réalité antique? », dans C. Nicolet (éd.), Du pouvoir dans l'Antiquité : mots et réalités, Genève, Droz, 1990, p. 53-67.

LOPEZ-RABATEL Liliane, MATHÉ Virginie \& MORETTI Jean-Charles (éd.), Dire la ville en grec aux époques antique et byzantine (Actes du colloque de Créteil, 10-11 juin 2016), Lyon, MOM Éditions, à paraître.

LOMBARDO Mario, « La documentazione epigrafica », dans Problemi della chora coloniale dall'Occidente al mar Nero (Atti del convegno di studi sulla Magna Grecia XL), Tarente, Istituto per la Storia e l'Archeologia della Magna Grecia, 2000, p. 73-114. 
MA John, « La cité grecque et les transferts culturels », DHA, 40 (1), 2014, p. 257-275.

MASTROCINQUE Attilio, « Roma quadrata », MEFRA, 110 (2), 1998, p. 681-697.

MATHÉ Virginie, Jean-Charles MORETTI \& Liliane RABATEL « Les mots grecs de la ville antique d'après l'Onomasticon de Pollux », Histoire urbaine, 42, 2015, p. 163-177.

MÉNARD Hélène \& PLANA-MALLART Rosa (éd.), Espaces urbains et périurbains dans le monde méditerranéen antique, Montpellier, Presses universitaires de la Méditerranée, coll. « Mondes anciens », 2015.

MOMIGLIANO Arnaldo, Problèmes d'historiographie ancienne et moderne, trad. fr., Paris, Gallimard, 1983.

MUSTI Domenico, Lo scudo di Achille: idee e forme di città nel mondo antico, Rome, Laterza, 2008.

NICOLET Claude, Le métier de citoyen dans la Rome républicaine, Paris, Gallimard, 1976.

NIPPEL Wilfried, «Finley and Weber. Some Comments and Theses », dans La Cité antique ? À partir de l'œuvre de M. I. Finley, Opus. Rivista internazionale per la storia economica e sociale dell'antichità, 6-8, 1987-1989, p. 43-50.

NIZZO Valentino, «L'idea della "città" alle radici della "Storia". Sociologia del confronto fra mondo indigeno peninsulare e mondo egeo all'alba della "colonizzazione": metodi, problemi e prospettive », dans M. Santucci, S. Sanchirico \& F. Pignataro (éd.), Ploutos \& polis: aspetti del rapporto tra economia e politica nel mondo greco (Atti del convegno internazionale di studi, Museo Nazionale Etrusco di Villa Giulia, 20-22 maggio 2013), Rome, E.S.S. Editorial Service System, 2016, p. 85-155.

OSANNA Massimo (dir.), Verso la città: forme insediative in Lucania e nel mondo italico fra IV e III sec. a.C. (Atti delle giornate di studio), Venosa, Osanna Edizioni, 2009.

OSBORNE Robin \& CUNLIFFE Barry (éd.), Mediterranean Urbanisation: 800-600 BC, Oxford, Oxford University Press, 2005.

PACCIARELLI Marco, Dal villaggio alla città: la svolta protourbana del 1000 a.C. nell'Italia tirrenica, Florence, All'insegna del Giglio, 2001.

PACCIARELLI Marco, «Verso i centri protourbani. Situazioni a confronto da Etruria meridionale, Campania e Calabria », Scienze dell'Antichità: storia archeologia antropologia, 15, 2009, p. 371-416.

PACCIARELLI Marco, « The Earliest Processes toward City-States, Political Power and Social Stratification in Middle Tyrrhenian Italy », Origini. Preistoria e protostoria delle civiltà antiche, XXXIX, 2016, p. 169-206.

PIÉRART Marcel, Platon et la cité grecque. Théorie et réalité dans la constitution des « Lois », Mémoires de la classe des lettres, tome LXII, fascicule 3, Bruxelles, Académie royale de Belgique, 1974.

PION Patrick, « Sortir des oppida », dans M.-C. Belarte \& R. Plana-Mallart (éd.), Le paysage périurbain en Méditerranée occidentale pendant la Protohistoire et l'Antiquité, Tarragone, Institut català d'arqueologia clàssica (Documenta 26), 2012, p. 47-56.

POLIGNAC François de, La Naissance de la cité grecque. Cultes, espace et société, VIII -VII ${ }^{e}$ siècles, Paris, La Découverte, 1995 (1984).

POLLINI Airton, « Le voisin mal-aimé : cité idéale et frontière en Grèce classique ", dans A. Koukoutsaki-Monnier (éd.), Représentations du transfrontalier (Actes $n^{\circ} 12$ ), Nancy, Presses universitaires de Nancy, 2011, p. 249-261. 
POLLINI Airton, « La ville grecque idéale : expériences concrètes et réélaborations », dans M. Chopin \& F. D'Antonio (éd.), Théâtralisation de l'espace urbain, Paris, Orizons, 2017, p. 97-120. PRADEAU Jean-François, Platon et la cité, Paris, PUF, 1997.

PUGLIESE CARRATELLI Giovanni, « Dalla "polis" all'“urbs” », dans Principi e forme della città, Milan, Libri Scheiwiller, 1993, p. 3-43.

REDDÉ Michel, « Les capitales des cités gauloises, simulacra Romae », dans M. Reddé \& W. Van Andringa (éd.), La naissance des capitales de cités en Gaule chevelue, revue Gallia, 72 (1), Paris, CNRS Éditions, 2015, p. 1-16.

RENDELI Marco (éd.), Le città visibili: archeologia dei processi di formazione urbana, vol. 1 : Penisola italiana e Sardegna, Rome, Officina Edizioni, 2015.

RESCIGNO Carlo \& SIRANO Francesco (éd.), Immaginando città: racconti di fondazioni mitiche, forma e funzioni delle città campane, Naples, Prismi, 2014.

RODRIGUEZ-ALMEIDA Emilio, Formae urbis antiquae. Le mappe marmoree di Roma tra la Repubblica e Settimio Severo, Rome, École française de Rome, 2002. Disponible en ligne sur <https://doi.org/ 10.4000/books.efr.1886>.

SAKELLARIOU Michaïl V., The Polis-State: Definition and Origin, Athènes, Research Centre for Greek and Roman Antiquity, 1989.

SMITH Michael, « How Can Archaeologists Identify Early Cities? Definitions, Types, and Attributes », dans M. Fernández-Götz \& D. Krausse (éd.), Eurasia at the Dawn of History: Urbanization and Social Change, Cambridge, Cambridge University Press, 2017, p. 153-168.

SNODGRASS Anthony, "Archaeology and the Study of the Greek City ", dans A. Snodgrass, Archaeology and the Emergence of Greece, Ithaca (NY), Cornell University Press, 2006 (1991), p. 269-289.

TARPIN Michel, «Oppidum, vu par les Romains... », dans Les Gaulois sont dans la ville : place de l'âge du fer dans l'archéologie urbaine (Actes du XXXII ${ }^{\mathrm{e}}$ Colloque international de l'AFEAF, Bourges, 30 avril-4 mai 2008), RACF, suppl. 35, 2009, p. 183-198.

TILBURG Cornelis R. van, Streets and Streams: Health Conditions and City Planning in the Graeco-Roman World, Leiden, Primavera Press, 2015.

VALLET Georges, « La cité et son territoire dans les colonies grecques d'Occident », dans La città e il suo territorio (Atti del convegno di studi sulla Magna Grecia VII), Tarente, Istituto per la Storia e l'Archeologia della Magna Grecia, 1967, p. 67-142.

VAN ANDRINGA William, « Le cens, l'autel et la ville chef-lieu : Auguste et l'urbanisation des Trois Gaules ", dans M. Reddé \& W. Van Andringa (éd.), La naissance des capitales de cités en Gaule chevelue, revue Gallia, 72 (1), Paris, CNRS Éditions, 2015, p. 17-33.

VLASSOPOULOS Kostas, Unthinking the Greek Polis: Ancient Greek History beyond Eurocentrism, Cambridge, Cambridge University Press, 2007.

VLASSOPOULOS Kostas, « Historicising the Closed City », dans M. Dana \& I. Savalli-Lestrade (éd.), La cité interconnectée dans le monde gréco-romain, IV siècle a.C.-IV siècle p.C. : transferts et réseaux institutionnels, religieux et culturels aux époques hellénistique et impériale, Bordeaux, Ausonius, 2019, p. 43-57.

WHITE Bob, « Interculturalité », dans Anthropen.org, Paris, Éditions des archives contemporaines, 2018. Disponible en ligne sur <https://doi.org/10.17184/eac.anthropen.082>. 
WHITLEY James, The Archaeology of Ancient Greece, Cambridge, Cambridge University Press, 2001.

ZURBACH Julien, « La formation des cités grecques : statuts, classes et systèmes fonciers », Annales HSS, 68 (4), 2013, p. 957-998.

\section{NOTES}

1. Avec le soutien de NovaTris, Centre de compétences transfrontalières (ANR-11-IDFI-0005) de l'Université de Haute-Alsace. Un premier atelier a eu lieu à Dijon le 4 mai 2018: "Nommer la ville. Vocabulaire de la cité et formes urbaines antiques». En abordant par des études de cas les différentes manifestations historiques de la cité et de la ville antiques, nous avons pu définir le questionnement destiné à constituer une trame initiale des travaux à entreprendre sur les vocabulaires et les définitions des formes urbaines antiques. Un deuxième atelier s'est tenu, toujours à Dijon, le 8 novembre 2018, « Naissance des cités : à l'aube des premières expériences urbaines». Nous nous sommes alors questionnés sur les prémices et l'émergence des phénomènes urbains - dans le monde égéen, en Italie et en Gaule - un domaine où les fouilles et les réflexions récentes ont apporté des données importantes autorisant un nouveau bilan sur les concepts de pré-urbanisme ou de proto-urbanisme.

2. L'Orient ne fait pas l'objet d'une analyse spécifique dans notre dossier. Il s'agit d'un choix au sein de notre démarche comparatiste qui a davantage visé, dans les premières étapes de notre travail, la perspective occidentale, strictement en lien avec nos recherches principales, et conformément aux préconisations de G. Pugliese Carratelli «Ma delle antiche città orientali ̀̀ totalmente diversa la polis per genesi, organizzazione e spirito animatore » $(1993,3)$. Nous n'excluons pas d'intégrer, dans les développements ultérieurs de ce projet de recherche, des phénomènes analogues à l'échelle méditerranéenne, en incluant notamment l'Orient en tant que tel et en donnant toute leur place à d'autres zones et processus historiques méditerranéens. Pour une approche comparatiste plus ambitieuse, voir Fernández-Götz \& Krausse (2016). Cette démarche comparatiste se retrouvait déjà dans Huot (1988), où les fondations ou formations de nouvelles villes en Orient et en Occident étaient mises en parallèle.

3. Smith (2017) propose d'en individualiser les caractéristiques archéologiques.

4. Adhérant à la tradition de la revue Gaia qui accueille ce dossier thématique, nous avons fait le choix d'insérer la traduction (par F. Létoublon) de l'article de K. J. Hölkeskamp (2002) qui, à partir du point de vue de l'anthropologie de la mémoire et de la tradition épique en tant que media les plus importants de la «mémoire culturelle» panhellénique, avait permis de porter un regard neuf sur l'émergence de la polis en esquissant de façon originale une sorte d' " archéologie de la polis », envisagée aussi bien au prisme des institutions que de son ancrage territorial et spatial. Le choix de certains espaces urbains comme représentation métonymique de la ville fait référence à un aspect précis, celui de l'organisation sociale et politique de la communauté, qui se reflète concrètement dans la définition progressive et dans l'«institutionnalisation » des espaces publics.

5. Fustel de Coulanges (1984 [1864], 151). Voir la relecture critique proposée par Momigliano (1983, «La Cité antique de Fustel de Coulanges », 402-423) et, plus récemment, Darcos, Hartog \& Manent (2015).

6. Voir la conclusion de ce dossier par M. T. Schettino. Voir aussi Carsana \& Schettino (2008), Coudry \& Schettino (2015) ; en dernier Caliò (2018) et Carsana \& Zizza (2019).

7. La bibliographie à ce propos est immense; on signale ici seulement quelques références spécifiques : Piérart (1974), Fantasia (1975), Pradeau (1997), Bertrand (2005) ; voir également nos propres contributions à ce sujet : Pollini (2011 et 2017). En effet, les Lois de Platon et la Politique d'Aristote condensent un savoir théorique bien plus étendu. Sur l'interaction entre 
aménagement de l'espace et organisation politique, il est essentiel de reconnaître le rôle majeur des cercles hippocratiques, notamment l'influence du traité Airs, eaux, lieux, ainsi que les analyses sur la météorologie : pour ne citer qu'un volume récent, voir Tilburg (2015).

8. A. Duplouy (2019) a ainsi récemment proposé une approche de sociologie historique et a envisagé la polis comme le produit de mécanismes complexes d'inclusion des uns et d'exclusion des autres: la cité grecque est une communauté d'initiés qui définissent et transmettent des comportements communs, non pas le produit d'une pensée rationnelle, ni une entité délibérément planifiée.

9. Voir notamment les réflexions méthodologiques dans Osborne \& Cunliffe (2005) et également le compte rendu par Étienne (2007).

10. Voir en particulier pour le cas de la cité grecque, point de départ de notre projet: Gawantka (1985), Sakellariou (1989) et, plus récemment, Caliò (2012, 23 et suiv.), Zurbach (2013), Greco (2016, 68-73), Greco (2018, 31-33). Pour une mise en perspective critique de l'historiographie traditionnelle notamment à la lumière des fouilles récentes, voir Davies (2016, 16-22). Voir aussi Nizzo (2016).

11. Max Weber, Die Stadt, écrit en 1911-1912, mais publié dans le deuxième tome d'Économie et société (Wirtschaft und Gesellschaft), publication posthume de 1921, traduction française La ville, 1982. Pour une vision critique, voir en particulier Finley $(1981,1985)$, Bruhns (1987-1989), Bruhns \& Nippel (1987-1989) et plus récemment Giangiulio (2004).

12. Gustave Glotz, La cité grecque : le développement des institutions, publié en 1928.

13. Voir Bruhns \& Nippel (1987-1989), Nippel (1987-1989). Voir le volume complet: Andreau et al. (1987-1989).

14. Ehrenberg (1937). Voir Whitley $(2001,165)$ et Davies $(2016,13-16)$.

15. Polignac (1995 [1984]). Les questions sur la pertinence même du concept de cité (polis) dans les écrits antiques et dans l'historiographie moderne ont été posées aussi par Gawantka (1985) et Sakellariou (1989).

16. Pour ce qui nous intéresse, voir notamment Hansen $(1993,1997 a, 1997 b, 2001)$ et l'aboutissement majeur du travail du Centre, son inventaire de cités grecques: Hansen \& Nielsen (2004). Voir également Fröhlich (2010).

17. L'article de A. Snodgrass (2006, publié à l'origine en 1991), s'interroge sur nos méthodes pour cerner la visibilité sur le terrain des transformations politiques et sociales au moment de l'« émergence des cités ».

18. Greco (1999) ; voir également Greco (2018).

19. Greco $(2016,67)$.

20. Pour une analyse, voir Zurbach (2013).

21. On signalera à ce titre l'ambition scientifique du colloque « Schémata. La città oltre la forma. Per una nuova definizione dei paesaggi urbani e delle loro funzioni », qui s'est tenu à Syracuse les 26-28 février 2020.

22. Corbier (2013).

23. Carlo Ginzburg (2012) a justement actualisé et approfondi les analyses sur le rôle de l'anachronisme inhérent à la recherche historique : « [Car,] au grand désespoir des historiens, les hommes n'ont pas coutume, chaque fois qu'ils changent de mœurs, de changer de vocabulaire. » (Bloch, 2013 [1949], 57)

24. Favory (2012). Voir également l'analyse de Leveau (2013).

25. Lévy (1990, 53-54).

26. Mathé, Moretti \& Rabatel $(2015,168)$.

27. Caliò $(2016,34)$.

28. Pugliese Carratelli (1993).

29. Lévy (1990).

30. Crifò $(2004,25)$. Sur politeia et ciuitas, voir également Nicolet (1976, chap. 1). 
31. Notamment dans la deuxième partie de ce dossier, avec les contributions de S. Lefebvre, S. Marcos, C. Stein et A.-F. Baroni.

32. Voir à ce titre la contribution de $\mathrm{C}$. Joncheray sur le monde étrusque.

33. D. Briquel (2008), en interrogeant le rôle des Étrusques dans l'évaluation du phénomène d'urbanisation de Rome, montre ainsi que pour les Romains il n'y avait de références possibles qu'à la culture grecque. Rome ne s'auto-perçoit pas comme ville étrusque.

34. Bourdin, Paoli \& Reltgen-Tallon $(2015,9)$. Sur les plans urbains de la Rome antique, les Formae urbis antiquae, voir Rodríguez-Almeida (2002).

35. Voir le commentaire dans Musti (2008, 3-13).

36. Voir Grandazzi (1993), Mastrocinque (1998), Carandini (2006), Coarelli (2012, 145-160).

37. Humm (2017). Pour une analyse comparative entre Athènes et Rome à l'époque de leur formation, voir Greco (2005) ; en particulier sur le rôle des espaces publics, voir Hölscher (2005).

38. Bourdin, Paoli \& Reltgen-Tallon $(2015,9)$.

39. Corbier (2013).

40. L'emploi abusif du mot uicus a, quant à lui, compliqué l'approche des agglomérations en évacuant les formes non urbaines : voir Favory (2012), avec bibliographie précédente.

41. Voir à ce propos l'article de G. Larguinat-Turbatte sur la constitution d'un paysage urbain de type grec et celui de D. Chatzivasiliou sur la Locride de l'Ouest et la cité de Naupacte.

42. "[...] polis cannot simply be rendered as 'city'. Polis is rather a human community, located in a specific place, and yet able to move, migrate and resettle while still remaining a polis. Thucydides says as much, when he suggests that soldiers make a polis wherever they encamp, because "men make the polis, not walls or a fleet of crewless ships". Equally there is a danger in the privileging of the urban elements of the polis, even when established on a particular site. A polis would necessarily have some rural areas, because of a need to feed its inhabitants, demands of security, and through the establishment of certain shrines outside the walls [...]. There is therefore a danger in reducing polis to city, not least because of the exclusion of the countryside [...], but also because it allows a too easy equation of forms of citizenship to urban formations. » (Elden, 2005, 355-359)

43. Voir par exemple dans ce dossier les contributions de É. Gomez \& D. Ugolini, D. Chatzivasiliou et V. Tosti.

44. Garcia (2015).

45. Voir les contributions de A. Fochesato, É. Gomez \& D. Ugolini, C. Joncheray et F. Quondam.

46. Voir Casevitz (1998).

47. Sur ce mot, les actes du colloque de Créteil «Dire la ville en grec aux époques antique et byzantine» (10-11 juin 2016) seront sans doute fondamentaux, cf. Lopez-Rabatel, Mathé \& Moretti (à paraître).

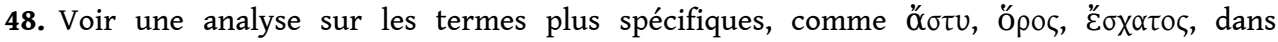
Bouchet (2012).

49. Cette conception de l'espace d'influence de la cité au-delà de son territoire civique a été proposée notamment dans les contributions de G. Vallet et E. Lepore dans les actes du colloque de Tarente de 1967 : Lepore (1967) et Vallet (1967). La définition de la chôra en tant que zone de pouvoir a été ensuite discutée et nuancée par E. Lepore (1973) lui-même, ainsi que par M. Lombardo (2000). L'analyse des témoignages épigraphiques menée par M. Lombardo ne montre aucun témoignage où la notion de $\chi \omega ́ p \alpha$ serait utilisée comme zone d'influence.

50. Larguinat-Tourbatte (2018).

51. Étienne (2013). Sur la notion de périurbain, voir en particulier Belarte \& Plana-Mallart (2012), Ménard \& Plana-Mallart (2015) et Finocchietti \& Lubtchansky (à paraître).

52. Bouchet (2015).

53. Voir les contributions de D. Lefèvre-Novaro et V. Tosti dans ce dossier.

54. En alternative au terme "proto-urbain", pour les $\mathrm{VI}^{\mathrm{e}}$ et $\mathrm{V}^{\mathrm{e}}$ siècles av. J.-C., P. Brun et B. Chaume proposent celui de "site à l'urbanisation inachevée » à partir de trois cas d'étude : le 
cas de la Heuneburg, celui de Vix et celui de Burges. «L'état actuel des connaissances permet de conclure qu'une tentative d'urbanisation s'est alors produite au nord des Alpes, de l'ouest de la Bavière à l'est du Berry au moins, un essai inachevé pour des raisons encore mal cernées, mais vraisemblablement d'ordre systémique et d'échelle continentale. Progresser dans la compréhension de cette éphémère tentative nécessite un radical changement d'échelle des opérations de terrain. Lui seul a permis les avancées réalisées ces dix dernières années, rendant, au passage, très improbable l'hypothèse selon laquelle les résidences princières auraient été dépourvues d'emprise territoriale. » (Brun \& Chaume, 2013, 341)

55. Cazanove \& Bourdin $(2019,331)$.

56. Voir les études de Pacciarelli (2001, 2009, 2016) ; voir aussi Osanna (2009), Rendeli (2015). En Italie centrale, le cas de Rome, "perhaps the first city-state in the western Mediterranean " (Fulminante, 2014, 251), est bien sûr d'une importance primordiale, mais sa croissance est conforme aux processus généraux de croissance menant à la formation autonome de centres urbains : voir Attema, Seubers \& Willemsen (2016). D'après les différents auteurs, ces développements ne doivent rien, ou presque, à des influences ou à des modèles étrangers.

57. Voir à ce propos les contributions de F. Quondam et A. Fochesato dans ce dossier.

58. Bourdin (2015).

59. Voir l'article de A. Fochesato dans ce volume.

60. Fernández-Götz (2018).

61. Tarpin (2009).

62. Pion (2015). Voir les contributions de A. Fochesato et S. Lefebvre.

63. Voir le bilan dans Barral, Coquet \& Nouvel (2012).

64. Voir Pierre Gros $(1998,11-12)$, qui invite à déplacer l'attention des chercheurs des « querelles terminologiques » au fonctionnement réel de ces agglomérations.

65. Voir la contribution de A. Fochesato dans ce dossier.

66. Van Andringa (2015).

67. Hanoune (2007).

68. Fichtl (2005 et 2013).

69. Reddé (2015).

70. Voir à cet égard les remarques de M. Rendeli à l'issue des travaux publiés dans le volume Le città visibili $(2015,9):$ : [...] la non esclusività del fenomeno urbano da parte di civiltà apparentemente più avanzate quanto piuttosto una molteplicità di esperienze in diverse aree del Mediterraneo, non sincroniche ma avvenute in un lasso di tempo piuttosto lungo se intendiamo il mare chiuso nel suo complesso, ma anche veramente breve se si considera la portata delle trasformazioni occorse nelle civiltà che vi si specchiano. La seconda è quella della assenza di costanti "reali": ciò a mio avviso rafforza l'idea che si tratti di esperienze endogene, parallele, che hanno come comune denominatore il cambiamento, inteso nel senso di trasformazioni profonde nella sfera della organizzazione, del modello economico, della complessità sociale. Il risultato appare comunque simile, ovvero l'intraprendere un percorso di costruzione di una propria autonomia e identità politica. » Voir également Garcia (2013).

71. Voir White (2018).

72. Ma (2014).

73. Voir à cet égard la session «Urbanization in Iberia and Mediterranean Gaul in the first millennium $\mathrm{BC} », \mathrm{du}$ 24th Annual Meeting of the European Association of Archaeologists, Barcelone : Belarte, Noguera, Plana-Mallart \& Sanmartí (à paraître).

74. Pour ce qui relève de la cité grecque, voir les travaux de K. Vlassopoulos, notamment Vlassopoulos (2007 et 2019). Précédemment, les travaux de Bernal $(1987,1991,2006)$ avaient déjà questionné certaines idées reçues de l'historiographie classique; voir aussi les nombreuses critiques qui lui ont été adressées: Lefkowitz \& MacLean Rogers (1996); et ses réponses: Bernal (2001). 


\section{RÉSUMÉS}

Comment envisager la recherche sur la cité antique? Quels sont les instruments les plus appropriés pour l'analyser? Quels sont les rapports entre l'émergence de la cité en tant que communauté indépendante et l'urbanisation telle que l'archéologie peut la restituer? Sans prétendre résoudre toutes ces questions difficiles, les contributions réunies dans ce dossier montrent que les définitions de la ville et de la cité antiques utilisées par les historiens et les archéologues contemporains sont loin d'être précisément fixées. Elles peuvent même varier selon les auteurs, les disciplines, les périodes et les contextes. Il n'y a donc pas de ville, mais des villes; pas de cité, mais des cités. L'enjeu d'une définition c'est aussi l'évaluation des théories, sous plusieurs aspects. Cet article introductif soulève ainsi un certain nombre d'interrogations qui ont servi de cadrage à notre programme de recherche dont les articles réunis dans ce dossier sont un premier résultat.

How to envisage research on the ancient city? What are the most appropriate instruments to analyse it? What are the relationships between the emergence of the city as an independent community and the urbanization as archaeology can restore it? Without claiming to resolve these difficult questions, the contributions gathered here show that urbanization and the definitions of the ancient city used by contemporary historians and archaeologists are far from being precisely determined; they can even vary according to the authors, disciplines, periods and contexts. As a consequence, there is no town, but towns; no city, but cities. The issue of a definition is also the evaluation of theories, in several aspects. Our introduction raises a number of questions which have served as a framework for our research program.

\section{INDEX}

Keywords : urbanisation, cities' formation, town, methodology, vocabulary

Mots-clés : urbanisation, formation des cités, ville, méthodologie, vocabulaire

\section{AUTEURS}

\section{ARIANNA ESPOSITO}

Université de Bourgogne

arianna.esposito@ubourgogne.fr

\section{AIRTON POLLINI}

Université de Haute-Alsace

airton.pollini@uha.fr 